\title{
Cytokine-Producing T Lymphocytes as a Marker of Prognosis and Rejection Episodes in Orthotopic Liver Transplantation
}

\author{
A. Paiva, S.V. Pereira, R. Ballesteros, A. Freitas, R. Perdigoto, O. Mota, J. Ferrão, L. Tomé, E. Furtado, \\ M.A. Cipriano, B. Geraldes, F.J. Oliveira, A.L. Furtado, and F.J. Regateiro
}

$\mathrm{H}^{\mathrm{B}}$ EPATIC allograft rejection results from the transplant recipient's immune response against allogeneic antigens of the donor liver. ${ }^{1}$ Acute cellular rejection (ACR) is common after orthotopic liver transplantation (OLT), usually occuring in the first 3 weeks after transplantation ${ }^{2}$ and is initiated by alloactivation of recipient $\mathrm{T}$ cells through both direct and indirect pathways. ${ }^{1}$ The control of ACR is one of the most important aspect in the treatment of posttransplant recipients because it determines prognosis. ${ }^{3}$

It is generally accepted that helper $\mathrm{T}$ lymphocytes play a central role in the triggering of allograft rejection, ${ }^{4}$ especially by the secretion of cytokines, that provide help for the activation, proliferation, and differentiation of other lymphocytes. ${ }^{1}$ After activation, helper $\mathrm{T}$ lymphocytes are polarized into two major subsets, Th1 and Th2, based on their cytokine production. $^{5-7}$ Similarly, cytotoxic T (Tc) lymphocytes may differentiate into Tc1 and Tc2 populations with cytokine profile analogous to those seen in Th subpopulations. $^{8}$

Th1 cells produce interleukin-2 (IL-2) and interferon- $\gamma$ $(\mathrm{IFN} \gamma)$ and promote immunopathologic functions by mediating delayed-type hypersensitivity (DTH), ${ }^{1}$ and frequently this type of immune activation is usually manifested during allograft rejection. ${ }^{6}$ Th 2 cells produce IL-4, IL-5 and IL-10, among others, ${ }^{5}$ provide B cell help, and could inhibit Th1 proliferation and cytokine secretion. Th2 cells may modulate acute rejection and the development of allograft tolerance. ${ }^{9}$ Also Th2 cytokine transcripts correlate with milder rejection episodes. ${ }^{10}$ These findings led to the hypothesis that immune deviation from a Th1 to a Th2 cytokine profile could play a crucial role in long-term graft survival. $^{6}$

Flow cytometry emerged as the premier technique for the enumeration of cytokine producing cells, allowing the simultaneous detection of at least two cytokines at single cell level.

The aim of this study was to quantify, by flow cytometry, the frequency of IFN $\gamma$, IL-4, and IL-10 producing T lymphocytes in the peripheral blood of patients undergoing OLT and correlate the results with the presence or absence of ACR. For this purpose, were studied patients at pretransplantation and twice a week in the first month after OLT.

\section{MATERIAL AND METHODS}

Twenty-two patients were studied immediately before OLT and two times a week in the first month after transplant. Five of these patients developed an ACR, confirmed by biopsy, between day 11 and 14 after transplant.

After density gradient separation of mononuclear cells, $2 \times 10^{6}$ cells were incubated in 24 wells plate (2 replicates), with $2 \mathrm{~mL}$ RPMI-1640 (Gibco; Paisley, Scotland) containing 10\% of human $\mathrm{AB}$ serum (Sigma, Germany), $50 \mathrm{ng} / \mathrm{mL}$ of phorbol 12-Myristate 13-Acetate (PMA; Sigma, Germany), $1 \mu \mathrm{mol}$ of ionomycin (Boehringer Mannheim, Germany) and $1.3 \mu \mathrm{L}$ of brefeldin A (Golgi plug-Pharmingen, San Diego, Calif) during four hours at $37^{\circ} \mathrm{C}$ in a humidified incubator in $5 \% \mathrm{CO}_{2}$ in air. Cultures in the absence of brefeldin A and/or PMA + ionomycin were also done to be used as negative controls.

Monoclonal antibodies (MoAbs), from R\&D Systems (Abing don, UK), were used anti-human cytokine MoAbs: anti-IFN $\gamma$ FITC/clone \#25723.11 and IL-4-PE/clone \#3007.11. Anti-human IL-10-PE/clone JES3-19F1 was obtained from Pharmingen. MoAb anti-human CD3 (PECy5 conjugated-TC) was obtained from Caltag laboratories (South San Francisco, Calif).

After cell surface staining of $1 \times 10^{6}$ mononuclear cells with 10 $\mu \mathrm{l}$ of anti-CD3-TC during 10 minutes at room temperature in the dark and wash twice with PBS, the intracellular cytokine staining was performed as described by Prussin and Metcalf. Thoroughly resuspended cells were previously fixed and permeabilized with 250 $\mu \mathrm{l}$ of cytofix/cytoperm (Pharmingen) solution during 20 minutes at $4^{\circ} \mathrm{C}$ in the dark. Then, cells were washed twice with $1 \times \mathrm{Perm} /$ wash solution (Pharmingen's cytostain Kit $10 \times$ solution-diluted to $1 \times$ in water) and pelleted. After an optimal concentration of MoAbs, specific for appropriate cytokines and isotype controls has been added, cells were incubated at $4^{\circ} \mathrm{C}$ for 30 minutes in the dark. Cells were washed twice with $1 \mathrm{~mL}$ of perm/wash solution and resuspended in $0.7 \mathrm{~mL}$ OS PBS (Gibco; Paisley, Scotland)-1\% paraformaldehyd (Merk Darnstadt, Germany).

Samples were acquired on the Epics XL (Coulter, Hialeah, Fla,

From the Histocompatibility Centre (A.A.P., S.V.P., A.F., F.J.R.) and the Liver Transplant Unit, Coimbra University Hospital (R.B., R.P., O.M., J.F., L.T., E.F., L.G., B.G., F.J.O., F.J.R.), Coimbra, Portugal, Pathology Department, Coimbra University Hospital, Coimbra, Portugal.

Supported by a grant C.F.I.C.S. 236/99 from Health Minister. Address reprint requests to Artur Augusto Paiva, Centro Histocompatibilidade do Centro, Faculdade de Medicina, 3040 Coimbra, Portugal. 


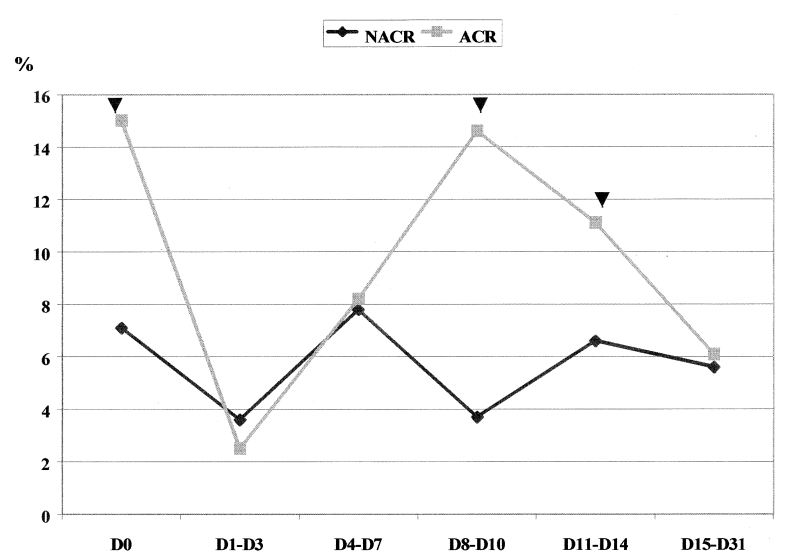

Fig 1. Profile of the frequency (\%) of IFN $\gamma$-producing $\mathrm{T}$ lymphocytes immediately before and after OLT in the group with acute cellular rejection (ACR) between days 11 and 14 and in the group without rejection episodes (NACR). $\mathbf{\nabla}$ Statistically significant value $(P<.05)$.

USA) using XL-II software. The number of events acquired for each sample was 15.000 on a $\mathrm{CD}^{+}$gate, after a first gate was performed on viable cells.

Statistical analysis were carried out using standard $t$ test performed by Macintosh (Cupertino, Calif) computer program Statview $512^{+}$(Abacus, Berkeley, Calif).

\section{RESULTS}

Immediately before OLT were observed in the group that developed an ACR between day 11 and 14, a higher frequency of IFN $\gamma$ producing T Ly (Fig 1) and a lower frequency of IL-4 and IL-10 producing T Ly, which results in a higher ratio Th1/Th2 in the group with ACR (Table 1).

After OLT were found in both groups (between day 1 and 3), a significantly decrease in the frequency of IFN $\gamma$ producing $\mathrm{T}$ Ly, which was more evident in the group with ACR (Fig 1). In the group without ACR, between day 4 and day 7 , this frequency returns to the level obtained before transplantation and remains without any significant change during the first month after transplantation (Fig 1).

In the group with ACR were observed, after day 3, a significant increase in frequency of IFN $\gamma$ producing $\mathrm{T} \mathrm{Ly}$, reaching the maximum value around day 10 (before the occurence of rejection episode between day 11 and 14) (Fig $1)$.

Although the observed variability of results obtained for the frequency of IL-10 and IL-4 producing T Ly, the group

Table 1. Frequency (\%) of Cytokine-Producing T-Ly

\begin{tabular}{lcccc}
\hline & IFN $\gamma$ & IL-4 & IL-10 & Th1/Th2 \\
\hline NACR $(n=17)$ & $7.1 \pm 6.6$ & $1.7 \pm 2.7$ & $1.0 \pm 0.7$ & $11.1 \pm 10.6$ \\
ACR $(n=5)$ & $15.0 \pm 11.1^{*}$ & $0.7 \pm 0.2$ & $0.4 \pm 0.2^{*}$ & $27.6 \pm 31.6^{*}$
\end{tabular}

*Statistically significant value $(P<.05)$.

NACR, no acute cellular rejection.

ACR, acute cellular rejection.

Frequencies obtained immediately before OLT. The results of Th1/Th2 were calculated by the ratio between IFN $\gamma$ and IL-4 values.

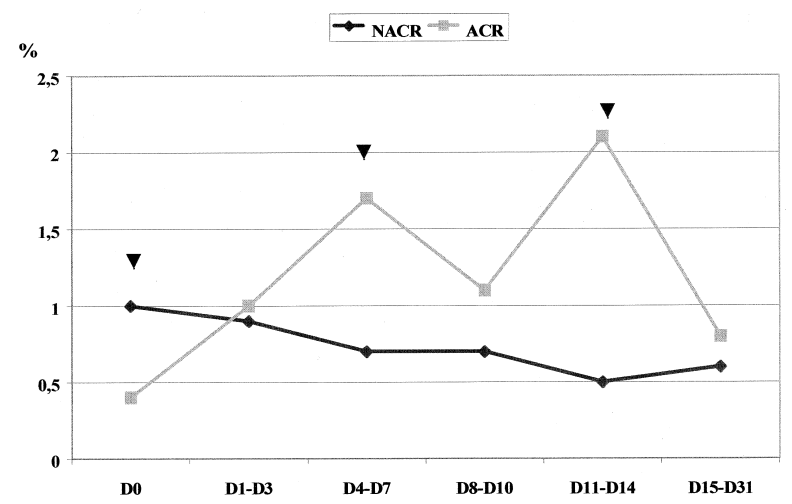

Fig 2. Profile of the frequency (\%) of $\mathrm{IL}-10$ producing $\mathrm{T}$ lymphocytes immediately before and after OLT in the group with acute cellular rejection (ACR) between days 11 and 14 and in the group without rejection episodes (NACR). $\boldsymbol{\nabla}$ Statistically significant value $(P<.05)$.

with ACR seems to have, after OLT, higher frequencies of IL-10 producing $\mathrm{T}$ Ly, presenting a maximum value between day 11 and 14 (Fig 2). Concerning the frequency of IL-4 producing T Ly, after OLT, we didn't find any significant statistical difference between the two studied groups (Fig 3).

\section{DISCUSSION}

Acute rejection of allografts remains a significant problem in clinical transplantation, decreasing the function and survival of transplanted organs. ${ }^{11}$ The severity of the rejection process may partially depend on the presence of environmentally primed $\mathrm{T}$ cells in the recipient that crossreact with donor histocompatibility antigens. ${ }^{12}$

Th1 type cytokines have been correlated with acute graft rejection severity as well as a kind of selective recruitment of Th1 cells into sites of DTH reactions, ${ }^{1}$ probably due to the expression of selectin ligands in cell surface. On con-

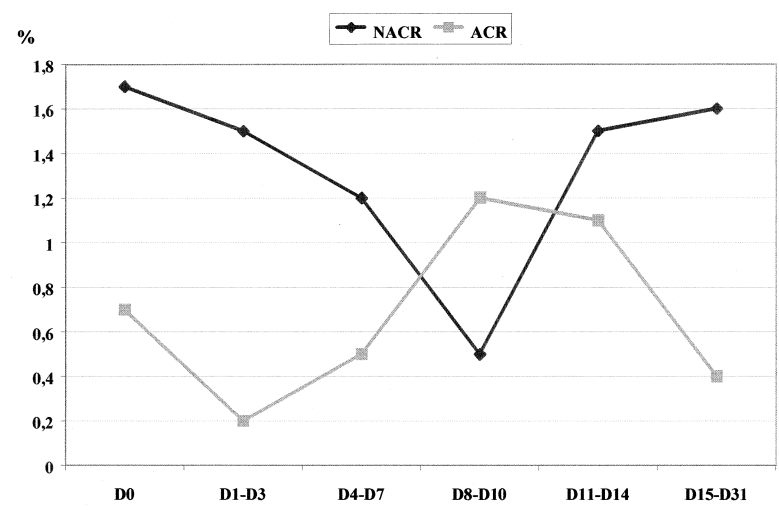

Fig 3. Profile of the frequency (\%) of IL-4 producing T lymphocytes immediately before and after OLT in the group with acute cellular rejection (ACR) between days 11 and 14 and in the group without rejection episodes (NACR). 
trary, Th2 type cytokines have been correlated to a certain tolerance induction and a promoting graft survival. ${ }^{13}$

The higher frequency of IFN $\gamma$ producing $\mathrm{T}$ Ly and of ratio Th1/Th2 observed immediately before OLT (Table 1) (Fig 1), in the group with ACR, seems to be related with a higher risk of posttransplantation rejection episodes and could be a good prognosis factor of liver transplantation. These findings are in agreement with others studies in renal transplantation. ${ }^{12}$

In the first days after OLT was observed, a significant decrease of frequency of IFN $\gamma$ and IL-4 producing T Ly, in both groups (Fig 1 and 3 ), probably due to the immunosuppression treatment. ${ }^{5}$ The frequency of IL-10 producing T Ly seems not be affected (Fig 2) since the transcription factor NFAT, inhibited by immunosuppressors, is not involved in IL-10 synthesis. ${ }^{5}$

Before and during the acute rejection episode, we observed a significant increase in the frequency of IFN $\gamma$ producing T Ly (Fig 1), pointing to a preferential activation, differentiation, and recruitment of Th1 cells, which are in agreement with the results obtained in other solid organ transplantation. ${ }^{10,14-16}$ This increase in frequency of IFN $\gamma$ producing T Ly, before and during ACR, could be an useful marker of prediction of rejection episodes in liver transplantation.

Although the low numbers of IL-10 and IL-4 producing T Ly obtained in both studied groups, the group with ACR seems to present higher frequencies of IL-10 producing cells, especially during the rejection episode (day 11 to 14) (Fig 2). A slight increase of IL-4 producing T Ly was also observed in this group before and during the rejection episodes (Fig 3). These small differences observed could be due to a differentiation of lower proportion of Ly to Th2 subset after activation of Th0 Ly. Nevertheless, higher levels of IL- $10^{15}$ and IL-10 high producer genotype recipients $^{13}$ have been correlated with graft rejection.

\section{REFERENCES}

1. Vierling JM: Liver Transpl Surg 5:S1, 1999

2. Bathgate AJ, Hynd P, Sommerville D, et al: Liver Transpl Surg 5:S479, 1999

3. Sakurai H, Mizuno S, Ohtakara K, et al: Transplant Proc 26:898, 1994

4. García-Alonso AM, Minguela A, Muro M, et al: Hum Immunol 53:64, 1997

5. van den Berg AP, Twihaar WN, Corver K, et al: Transplant Proc 30:2378, 1998

6. Strom TB, Roy-Chaudhury P, Manfro R, et al: Curr Opin Immunol 8:688, 1996

7. Mosmann TR and Sad S: Immunol Today 17:138, 1996

8. Mosmann TR, Li L, Hengartner H, et al: Ciba Found Symp 204:148, 1997

9. TaKeuchi T, Lowry RP, Konieczny B, et al: Transplantation 53:1281, 1992

10. Stinn JL, Taylor MK, Becker G, et al: Am J Pathol 153:1383, 1998

11. Koga S, Auerbach MB, Engeman TM, et al: J Immunol 163:4878, 1999

12. Heeger PS, Greenspan NS, Kuhlenshimdt S, et al: J Immunol 163:2267, 1999

13. Sankaran D, Asderakis A, Ashraf S, et al: Kidney International 56:281, 1999

14. Furukawa Y, Matsumori A, Hwang MW, et al: Jpn Circ J 63:775, 1999

15. Van Gelderen BE, Van Der Lelij A, Peek R, et al: Opthalmic Res 32:157, 2000

16. Diamond AS, Gill RG: J Immunol 165:247, 2000 